\title{
Non-re-cooling implantation of marginal liver graft after machine perfusion: report of a case
}

\author{
Weiqiang Ju ${ }^{1,2,3 \#}$, Zhitao Chen ${ }^{1,2,3 \#}$, Qiang Zhao ${ }^{1,2,3 \#}$, Yixi Zhang ${ }^{1,2,3}$, Changjun Huang $^{1,2,3}$, Linhe Wang ${ }^{1,2,3}$, \\ Caihui Zhu ${ }^{1,2,3}$, Yinghua Chen ${ }^{1,2,3}$, Zhiyong Guo ${ }^{1,2,3}$, Maogen Chen ${ }^{1,2,3}$, Xiaoshun $\mathrm{He}^{1,2,3}$ \\ ${ }^{1}$ Organ Transplant Center, First Affiliated Hospital of Sun Yat-sen University, Guangzhou, China; ${ }^{2}$ Guangdong Provincial Key Laboratory of Organ \\ Donation and Transplant Immunology, Guangzhou, China; ${ }^{3}$ Guangdong Provincial International Cooperation Base of Science and Technology \\ (Organ Transplantation), Guangzhou, China \\ \#These authors contributed equally to this work. \\ Correspondence to: Xiaoshun He; Maogen Chen; Zhiyong Guo. Organ Transplant Center Division, First Affiliated Hospital of Sun Yat-sen University, \\ Guangzhou, China. Email: gdtrc@163.com; chenmg3@mail.sysu.edu.cn; rockyucsf@126.com.
}

\begin{abstract}
Early allograft dysfunction (EAD), primary graft nonfunction (PNF) and biliary complications affect postoperative survival after liver transplantation (LT). Ischemia injury is one of the major factors affecting liver allograft functional recovery. Ischemia-free liver transplantation (IFLT) has obvious advantages for the recovery of allograft function and complication incidence compared with conventional procedures. However, its use is limited when the donor and the recipient are not in the same hospital and donors should be donor after brain death (DBD). We propose an approach to avoid double warm ischemic injury by implanting marginal donor liver directly by using normothermic machine perfusion (NMP) without recooling. Here, we report the first case of non-re-cooling implantation for marginal donor in LT. Donor liver biopsies before procurement showed 50\% macrovesicular steatosis, and the recipient was a 67-year-old man with decompensated cirrhosis secondary to a 21-year hepatitis B virus (HBV) infection. The donor liver was maintained by NMP without re-cooling before implantation. The highest levels of alanine transaminase (ALT) and aspartate transaminase (AST) after surgery were 235 and 1,076 U/L, respectively, on the first postoperative day (POD). The patient was discharged within 2 weeks and showed good recovery. Thus, it is feasible to use Non-re-cooling implantation for marginal donor in LT.
\end{abstract}

Keywords: Ischemia-free liver transplantation; normothermic machine perfusion; marginal donor liver; case report

Submitted Mar 23, 2020. Accepted for publication Sep 11, 2020.

doi: $10.21037 /$ atm-20-2774

View this article at: http://dx.doi.org/10.21037/atm-20-2774

\section{Introduction}

Due to the development of surgical technology and organ cold preservation technology and the use of immunosuppressants, liver transplantation (LT) has become the only effective way to treat end-stage liver diseases (1). However, the donor liver will inevitably experience warm ischemia, cold preservation, a second warm ischemia and reperfusion injury in the process of procurement, transportation and implantation. Ischemia-reperfusion injury (IRI) is one of the major reasons for early allograft dysfunction (EAD), primary graft nonfunction (PNF) and biliary complications after liver transplantation $(2,3)$. Cold preservation helps to reduce cellular physiological metabolism but fails to slow the destruction of cellular integrity (4). This may become more evident when using extended criteria donor livers.

For decades, efforts have been made to ameliorate IRI. The use of normothermic machine perfusion (NMP) technology (5) for organ preservation has progressed significantly and been proven to be effective in clinical trials (6). It can provide continuous oxygen and nutrient 

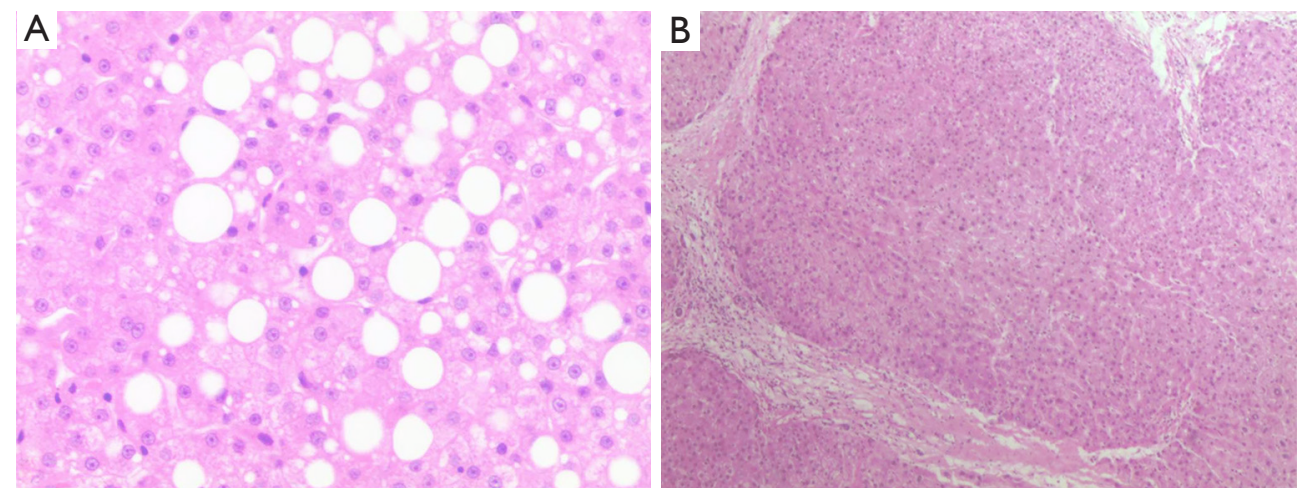

Figure 1 Liver tissue biopsies with H\&E staining before procurement (A) $(\times 50)$ and after reperfusion ( $\times 10)(\mathrm{B})$. (A) Inflammatory cells in the portal area were not obvious, and fatty degeneration of hepatocytes was found in the inner part of the liver parenchyma, accounting for approximately $50 \%$ steatosis. (B) Mild watery degeneration of hepatocytes and a small number of inflammatory cells were found in the portal area.

supply during perfusion (7). The use of whole blood as a perfusate is a common clinical practice. In traditional processes, the liver is transported in cold preservation and placed in the NMP device for at least 4 hours after procurement. Before implantation, the liver graft is usually flushed with cold solution. However, this may cause double IRI of the organ due to the additional experience of going from cold to warm and then from warm to cold compared with organs that are subjected to the regular cold preservation technique (8). Although there is an obvious advantage of NMP for cold preservation for organ renovation, the second experience of warm ischemia when using the NMP technique would be avoided by this technique. IFLT was designed to completely avoid ischemia injury during transplantation (9). The results of the first case of IFLT showed that this technique had obvious advantages for the recovery of allograft function and the reduction of complication incidence compared with the conventional procedure. Limitations of IFLT is that when the donor and the recipient are not in the same hospital. Here, we report the first case of the use of the NMP technique without double IRI injury of a marginal donor liver. We present the following article in accordance with the CARE reporting checklist (available at http://dx.doi.org/10.21037/atm-202774).

\section{Case presentation}

The recipient patient was a 67 -year-old man with a blood type of $\mathrm{B}+$ and decompensated cirrhosis secondary to a 21-year hepatitis B virus (HBV) infection, and his body mass index (BMI) was $19.9 \mathrm{~kg} / \mathrm{m}^{2}$. Preoperative computed tomography angiography (CTA) examination showed cirrhosis, moderate ascites, splenomegaly, esophageal and gastric varices. The aspartate transaminase (AST), alanine transaminase (ALT) and total bilirubin (Tbil) levels were $18 \mathrm{U} / \mathrm{L}, 33 \mathrm{U} / \mathrm{L}$, and $46.3 \mu \mathrm{mol} / \mathrm{L}$, respectively. The model for end-stage liver disease (MELD) score was 13 . There was no history of hypertension, diabetes or coronary heart disease before the operation, and pulmonary function was normal.

The donor was a 59-year-old woman with a blood type of $\mathrm{B}+$ and a BMI of $21.5 \mathrm{~kg} / \mathrm{m}^{2}$. The AST, ALT and Tbil levels were $19 \mathrm{U} / \mathrm{L}, 24 \mathrm{U} / \mathrm{L}$, and $23.1 \mu \mathrm{mol} / \mathrm{L}$, respectively. The following risk factors: hypernatremia (sodium, $153 \mathrm{mmol} / \mathrm{L}$ ), 50\% macrovesicular steatosis according to liver biopsies (Figure 1) and a foreseeable cold ischemia time of more than 10 hours; this conformed to a marginal donor liver. To minimize the risk of EAD or PNF, continuous NMP without re-cooling was performed for this patient.

All procedures followed were in accordance with the ethical standards of the responsible committee on human experimentation (institutional and national) and with the Helsinki Declaration of 1964 and later versions (as revised in 2013).Written informed consent was obtained from the patient for publication of this study and any accompanying images. A copy of the written consent is available for review by the Editor-in-Chief of this journal. The donor liver was procured using the standard cold perfusion technique, and the organ was preserved in static cold storage in University of Wisconsin (UW) solution. When the donor liver arrived, it was trimmed for insertion of a straight $24 \mathrm{Fr}$ cannula into the portal vein $(\mathrm{PV})$ and an $8 \mathrm{Fr}$ arterial cannula into splenic artery (SA) to allow the connection to the NMP machine, 


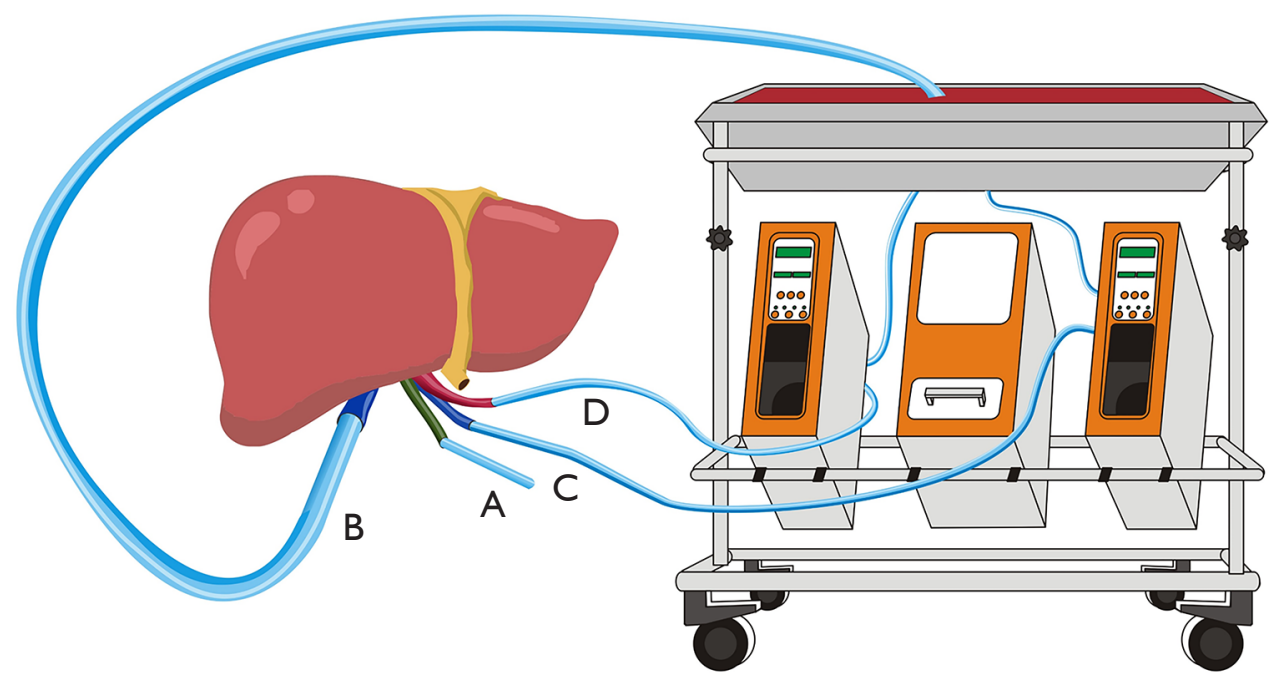

Figure 2 Diagram of the cannula location in NMP without re-cooling. (A) Tube placed in the common bile duct to drain bile. (B) Cannula placement into IHIVC to allow intrahepatic blood flow. (C) Cannula placement into PV. (D) Artery cannula placement into SA. NMP, normothermic machine perfusion.
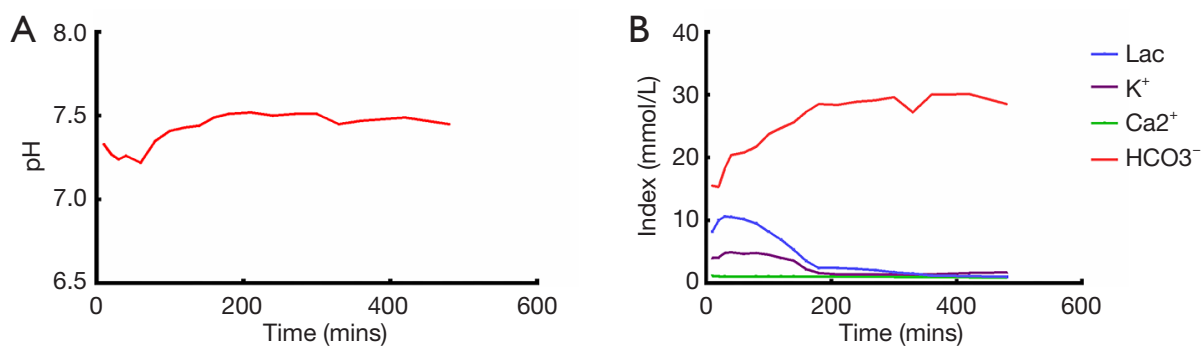

Figure 3 Changes in physicochemical indexes during perfusion. (A) Changes in pH during perfusion. (B) Changes in blood gas indexes during perfusion.

as previously reported (9). After a 9-hour 20-minute cold ischemia period, the donor liver was perfused with a Liver Assist Device (Organ Assist, Groningen, the Netherlands) at $37^{\circ} \mathrm{C}$. A drainage tube was placed into the common bile duct for bile detection (Figure 2). Before vascular anastomosis, a $34 \mathrm{Fr}$ caval cannula was placed into the infrahepatic inferior vena cava (IHIVC) to ensure blood flow to the organ reservoir during the anhepatic phase. Just before the NMP circuit was established, 10 units of washed red blood cells (WRBC) at $37{ }^{\circ} \mathrm{C}$ were used as the perfusate for circumfusion as previously reported.

NMP lasted for a total of 6 hours. During perfusion, the acid-base equilibrium and electrolyte balance were maintained throughout the perfusion process (Figure 3). The $\mathrm{pH}$ level was maintained at 7.45 by adding sodium bicarbonate. The lactate level and potassium level dropped significantly during perfusion from 10.5 to $1.6 \mathrm{mmol} / \mathrm{L}$ and 4.9 to $1.7 \mathrm{mmol} / \mathrm{L}$, respectively. The sodium and calcium levels remained stable during perfusion at 145 and $1.0 \mathrm{mmol} / \mathrm{L}$, respectively. The liver remained in the perfusion machine until implantation.

The liver implantation was performed similarly as that in IFLT. The donor liver was moved from the reservoir and placed in the recipient's abdominal cavity as NMP was nonstop. The donor SHIVC, HA and PV were anastomosed to the recipient counterparts in an end-to-end fashion using 3-0, 8-0, and 5-0 Prolene, respectively. NMP was stopped before the removal of $\mathrm{PV}$ cannula and the anastomosis of PV. After reperfusion, the donor IHIVC was then anastomosed to the recipient IHIVC. The donor common 

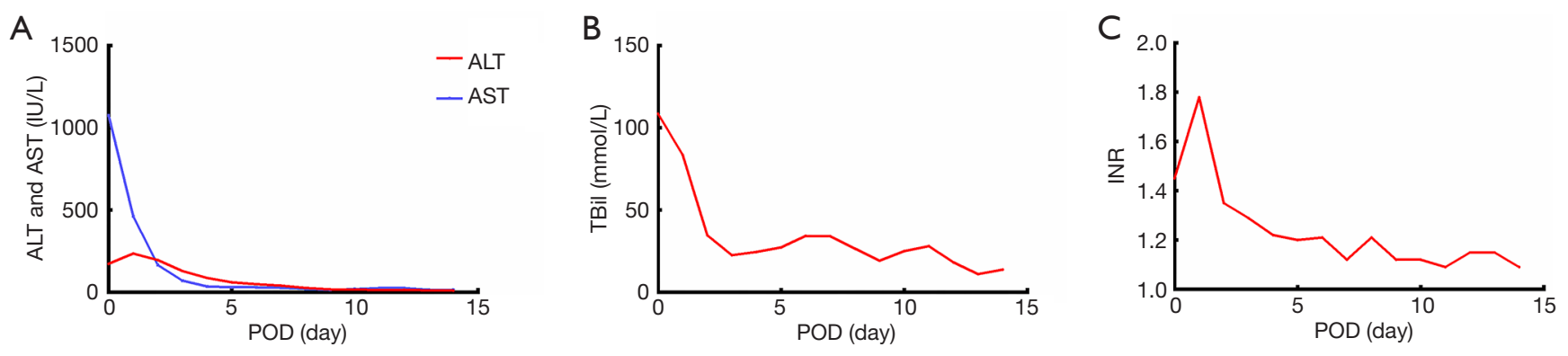

Figure 4 Postoperative liver function tests of the recipient. (A) Changes in AST and ALT; (B) changes in Tbil; (C) changes in INR. ALT, alanine transaminase; AST, aspartate transaminase; INR, international normalized ratios.
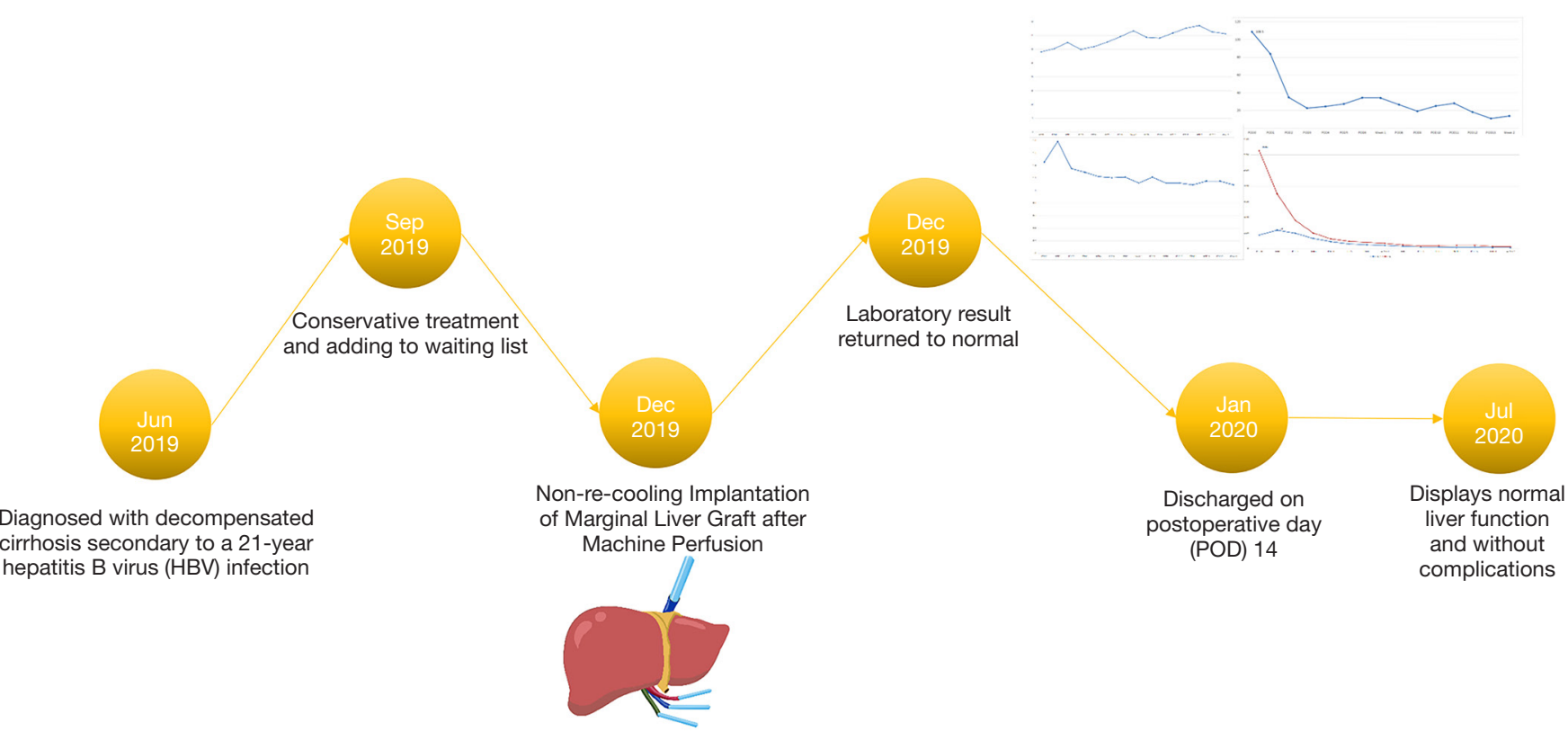

Figure 5 Timeline of diagnosis and treatment of patient.

bile duct was end-to-end anastomosed to the recipient common bile duct after withdrawal of the draining tube.

The anhepatic phase was 57 minutes. Blood loss was $1,800 \mathrm{~mL}$. Four units of RBCs and 8 units of fresh frozen plasma (FFP) were used during the operation. The total operation time was 365 minutes. The patient was extubated at 12 hours post-transplantation. The intensive care unit (ICU) stay time was 25 hours. The maximal ALT, AST and TBil were reached at postoperative day (POD) 0, 1 and 0 , respectively (Figure 4A,B). The international normalized ratios (INR) returned to normal on POD 4 (peak INR was 1.8 on POD1) (Figure 4C). After reperfusion, the donor liver biopsy after perfusion showed mild watery degeneration of the hepatocytes and infiltration of a small number of inflammatory cells into the portal area (Figure 1). Neither EAD nor PNF was presented. The patient was discharged on POD 14. By present, during the 6-month local followup, the patients recovered well without allograft rejection or vascular, biliary or other complications. Timeline of treatment was shown in Figure 5.

\section{Discussion}

LT is considered the only curative treatment for patients with end-stage liver diseases such as decompensated liver cirrhosis, acute liver failure and hepatocellular cancer. LT 


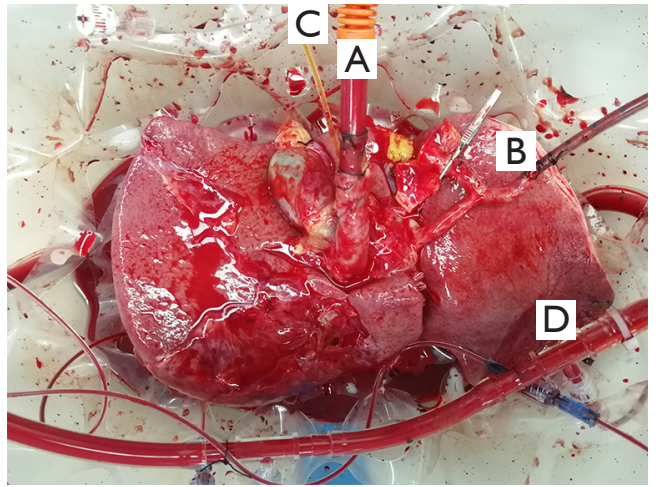

Figure 6 Preservation processes during NMP. (A) A straight $24 \mathrm{Fr}$ cannula into PV; (B) an 8 Fr arterial into splenic artery (SA); (C) A drainage tube into common bile duct; (D) a 34 Fr caval cannula into IHIVC. NMP, normothermic machine perfusion.

survival within one year has reached $80 \%$ to $90 \%$ due to progressive improvements in surgical methods, rejection treatment strategies and sepsis control (10). However, the main problems of conventional LT are that the donor liver will inevitably experience warm ischemia, cold preservation and reperfusion injury. Cold preservation helps to reduce cell physiological metabolism but fails to slow the destruction of cellular integrity. IRI is the main cause of EAD, PNF and biliary complications (11). Solving these problems can help to improve the postoperative survival rate and reduce postoperative complications. NMP is designed to ameliorate IRI in donor organs after cardiac death (DCD) or long cold ischemia time and has been proven to be effective. However, current NMP techniques are not perfect and have potential problems. Before implantation, the liver graft is usually flushed with cold solution, which may cause double IRI due to the rapid temperature change. To minimize the risk of EAD or PNF, we decided to perform continuous NMP without re-cooling for a marginal donor liver and obtained convincing outcomes. di Francesco et al. (12) has authored a case report on the feasibility of NMP with the avoidance of re-cooling. However, they do not address specific operations. To the best of our knowledge, this is the first reported case in which it was described how to avoid re-cooling before implantation. It is credible that implantation without re-cooling can help to potentially reduce the risk of $\mathrm{EAD}$ or PNF for marginal donor liver, according to our case report.

Marginal donor liver is a narrower definition than nonideal donor liver. It refers to a donor liver with an increased risk of PNF and EAD after transplantation (13). Research of Maring et al. has shown that the criteria of a marginal donor liver include an old donor liver (from a patient over 55 years old), a donor liver subjected to a long warm ischemia time, a donor liver with moderate to severe steatosis, and a donor liver that is HBsAg-positive (14). In this case report, the donor liver was obtained from a 59-year-old woman and showed 50\% macrovesicular steatosis and a long cold ischemia time, confirming that it was a marginal donor liver. It was not suitable for transplantation before functional restoration by machine perfusion. NMP without the re-cooling technique could provide a unique method for salvaging marginal donor organs, even after discarding the liver. The determination of whether this technique produces better outcomes than NMP alone needs further RCT data confirmation.

Amazingly, a new technique has been reported to ensure the complete avoidance of ischemia injury during transplantation, which is defined as IFLT. The donor liver is procured, preserved, and implanted under continuous NMP without a cold perfusion process. This technique has obvious advantages in the recovery of allograft function and the reduction of complication incidence compared with the conventional procedure (15). There have also been randomized clinical trials (RCTs) to confirm its feasibility (16). However, there are some limitations for IFLT. For a DCD donor liver, its application is limited because there is no way to establish effective donor liver circulation (17). In addition, because of the inconvenience of transportation with Liver Assist, the donor and the recipient should be in the same hospital. This technique requires further innovation. Compared with IFLT, this technique has its own unique advantages. First, it is suitable for a DCD donor liver and can tolerate long-distance transportation to ensure appropriate cold ischemia. Additionally, compared with those of IFLT, the techniques of procurement and perfusion are simpler and more widespread. Therefore, NMP without re-cooling can be a good supplement for IFLT that could both reduce the incidence of postoperative complications and increase the availability of marginal donor livers.

There are certain limitations for this technique. First, compared with those of conventional procedures, the preservation processes are still complex (Figure 6) and need to be further simplified in the future to shorten the cold ischemia time. In addition, the technology still needs to be tested by RCT to verify its effectiveness and feasibility. There is another disadvantage of our technique. The liver will still experience ischemia when we perform anastomosis 
of $\mathrm{PV}$. A possible optimization measure is to establish the collateral circulation of PV to safeguard the whole process of perfusion.

In conclusion, our results demonstrate that it is feasible to perform non-re-cooling implantation for marginal livers. Further reports of this technique are needed before achieving any promising results.

\section{Acknowledgments}

Funding: This work was supported by the National Natural Science Foundation of China (81401324 and 81770410), Guangdong Basic and Applied Basic Research Foundation (2020A1515011557), Science and Technology Planning Project of Guangdong Province (2016A020215048), Guangdong Provincial Key Laboratory of Organ Donation and Transplant Immunology (2013A061401007), Guangdong Provincial International Cooperation Base of Science and Technology (Organ Transplantation) (2015B050501002).

\section{Footnote}

Reporting Checklist: The authors have completed the CARE reporting checklist. Available at http://dx.doi.org/10.21037/ atm-20-2774

Conflicts of Interest: All authors have completed the ICMJE uniform disclosure form (available at http://dx.doi. org/10.21037/atm-20-2774). The authors have no conflicts of interest to declare.

Ethical Statement: The authors are accountable for all aspects of the work in ensuring that questions related to the accuracy or integrity of any part of the work are appropriately investigated and resolved. All procedures followed were in accordance with the ethical standards of the responsible committee on human experimentation (institutional and national) and with the Helsinki Declaration of 1964 and later versions (as revised in 2013). Written informed consent was obtained from the patient for publication of this study and any accompanying images.

Open Access Statement: This is an Open Access article distributed in accordance with the Creative Commons Attribution-NonCommercial-NoDerivs 4.0 International License (CC BY-NC-ND 4.0), which permits the noncommercial replication and distribution of the article with the strict proviso that no changes or edits are made and the original work is properly cited (including links to both the formal publication through the relevant DOI and the license). See: https://creativecommons.org/licenses/by-nc-nd/4.0/.

\section{References}

1. Morris PJ. Transplantation--a medical miracle of the 20th century. N Engl J Med 2004;351:2678-80.

2. Schlegel A, Dutkowski P. Impact of Machine Perfusion on Biliary Complications after Liver Transplantation. Int J Mol Sci 2018;19:3567.

3. Zhai Y, Petrowsky H, Hong JC, et al. Ischaemiareperfusion injury in liver transplantation--from bench to bedside. Nat Rev Gastroenterol Hepatol 2013;10:79-89.

4. Briceno J, Marchal T, Padillo J, et al. Influence of marginal donors on liver preservation injury. Transplantation 2002;74:522-6.

5. Karangwa SA, Dutkowski P, Fontes P, et al. Machine Perfusion of Donor Livers for Transplantation: A Proposal for Standardized Nomenclature and Reporting Guidelines. Am J Transplant 2016;16:2932-42.

6. di Francesco F, Pagano D, Martucci G, et al. Normothermic Machine Perfusion Using an Air/Oxygen Mixer for Reconditioning a Liver From a Marginal Brain Death Donor. Artif Organs 2017;41:E66-8.

7. Mergental H, Perera MT, Laing RW, et al. Transplantation of Declined Liver Allografts Following Normothermic ExSitu Evaluation. Am J Transplant 2016;16:3235-45.

8. Houben P, Manzini G, Kremer M, et al. Graft rinse prior to reperfusion in liver transplantation: literature review and online survey within the Eurotransplant community. Transpl Int 2015;28:1291-8.

9. He X, Guo Z, Zhao Q, et al. The first case of ischemiafree organ transplantation in humans: A proof of concept. Am J Transplant 2018;18:737-44.

10. Meirelles Junior RF, Salvalaggio P, Rezende MB, et al. Liver transplantation: history, outcomes and perspectives. Einstein (Sao Paulo) 2015;13:149-52.

11. de Rougemont O, Dutkowski P, Clavien PA. Biological modulation of liver ischemia-reperfusion injury. Curr Opin Organ Transplant 2010;15:183-9.

12. di Francesco F, Pagano D, Martucci G, et al. Normothermic Machine Perfusion in Liver Transplantation: Feasibility and Promise of Avoiding Recooling Before Engrafting. Liver Transpl 2019;25:1113-7.

13. Sass DA, Reich DJ. Liver transplantation in the 21 st 
century: expanding the donor options. Gastroenterol Clin North Am 2011;40:641-58.

14. Maring JK, Klompmaker IJ, Zwaveling JH, et al. Poor initial graft function after orthotopic liver transplantation: can it be predicted and does it affect outcome? An analysis of 125 adult primary transplantations. Clin Transplant 1997;11:373-9.

15. Zhao Q, Huang S, Wang D, et al. Does Ischemia Free Liver Procurement Under Normothermic Perfusion Benefit the Outcome of Liver Transplantation? Ann

Cite this article as: Ju W, Chen Z, Zhao Q, Zhang Y, Huang C, Wang L, Zhu C, Chen Y, Guo Z, Chen M, He X. Non-recooling implantation of marginal liver graft after machine perfusion: report of a case. Ann Transl Med 2020;8(21):1465. doi: 10.21037/atm-20-2774
Transplant 2018;23:258-67.

16. Huang C, Huang S, Tang Y, et al. Prospective, singlecentre, randomised controlled trial to evaluate the efficacy and safety of ischaemia-free liver transplantation (IFLT) in the treatment of end-stage liver disease. BMJ Open 2020;10:e035374.

17. Skwirczynska E, Wroblewski O, Serwin N, et al. Donors After Circulatory Death: Theme of Present or a Future? The Research in West Poland. Transplant Proc 2019;51:3199-204. 